\title{
A PLEA FOR THE EXTENDED USE OF UNNA'S PASTE BANDAGES
}

\author{
By HAROLD DODD, Ch.M., F.R.C.S. \\ (Surgeon, King George Hospital, Ilford; Surgeon, Royal Hospital, Richmond; and E.M.S, \\ Surgeon, London Homaeopathic Hospital).
}

An Unna's paste bandage has been conventional for the treatment of varicose eczema and ulceration for years. With the advent of elastoplast applied in the Dickson Wright method, its use, which was messy, has contracted, but it has continued to be used by those who knew its merits, and also as an alternative dressing for the appreciable percentage of patients intolerant to elastoplast. Thus Unna's paste maintains its usefulness although on a diminished scale.

The purpose of this article is to describe the use of Unna's paste on areas of the body other than the leg, and to make a plea for its wider adoption. It has been used steadily by the writer since 1938 and the accumulated experience confirms its effectiveness. It has been successful practically wherever it has been applied. Amongst its virtues is that of almost consistent acceptability to patients, it seldom irritates the skin like elastoplast, it is a good dressing for wounds of all description, the skin heals rapidly under it. It requires to be changed only at long intervals, repeatedly it has been on for four to twelve weeks. The semi-stiff semi-waterproof elastic dressing protects against soiling, buffers ordinary wear and tear, is a splint; and it keeps the part warm, the last being an overlooked therapeutic agent. Apart from promoting healing, it is time-saving and economically satisfactory. It has been applied beneficially to burns and scalds, to boils, carbuncles, whitlows, abscesses, granulating wounds, to ulcers, skin grafts and to contused indurated parts.

\section{The Unna bandage}

The type of application varies. The classical one is that of a jar of the warmed, melted paste which is brushed on to the part, alternating with layers of bandage. The writer dislikes this and prefers sterile bandages which are loosely rolled, and then simmered in the boiling melted paste until completely saturated. The hot bandages are lifted out on to a sterile dish to cool to body temperature before they can be put on. Nursing staffs quickly become adept at their preparation. A disadvantage of Unna's paste bandages is that notice of their use is required for preparation. It is conceded at the outset, that the method of application is messy and the patient, the dresser and the floor need to be protected from the paste which falls from the bandage; in hospital these points are easily managed. There is a certain satisfaction lingering from our early days in handling the "sloppy" medium. In private practice, an almost equally effective and more convenient preparation is available; it is that of viscopaste, cellanband or dalzoband. They are ready-made impregnated bandages which are tacky at room temperature. They are also obtainable with varying proportions of sulphanilamide and other salves.

\section{The method of application}

The method of application is that of a high standard of bandaging, aiming at a slight even tension throughout, without fold, without crease, and without twist. It is a task for the senior, until he has trained his assistants to give the full attention of craftsmanship necessary for the comfortable, durable and efficient bandage. Over the ulcer or lesion a slab of the bandage the size and thickness of a folded and ironed ladies' or gentleman's handkerchief is placed. Another point to be faithfully observed is that joints must be covered all round with longitudinal strips of three to four thicknesses of bandage. For instance, at the ankle an anterior strip is laid over the extensor tendons on the dorsum of the foot, ankle and lower leg, lateral lengths over the external and internal malleoli and a posterior piece over the tendo-achilles. If these are omitted, the bandge quickly develops hard transverse creases across the front and back of the ankle which cut the skin deeply, cause pain and laceration and require the bandage to be taken off in two to three days. So strongly does the writer feel the importance of the longitudinal strips that he teaches that unless they are put on, it is better to throw the bandage into the wastepaper basket immediately, and save the patient suffering and loss of confidence, and the doctor's or nurse's time. 


\section{The duration of use of the bandage}

The period during which the bandage is kept on varies with the lesion to which it is applied. It may require to be removed daily, later on alternate days, but invariably the period of changing becomes longer, until one may be kept on for three months. The infrequent changes save pain, time of incapacitation, of dressing and expense for the patient and hospital. The technique is, particularly advantageous in extensive burns, and the improvement which follows in the general condition, personality and morale of these patients is gratifying.

\section{Conditions to which the bandage has been applied}

The bandages have been applied effectively to the following conditions:

\section{The Scalp.}

Several patients with injuries of the scalp such as partial or total avulsion, burns, and skin grafts have been treated. When the lesion has been cleaned, it is powdered with sulphanilamide and viscopaste bandage is applied to the head and neck. The application is comfortable and ${ }_{0}$ healing has been progressive. Two to four weeks have been the usual period without change. 3

\section{Boils and Carbuncles on the neck.}

It is in the tiresome condition of recurrent boils and carbuncles of the back of the neck that Unna's paste bandage has been outstandingly successful. A slab is put on the lesion $\vec{N}$ itself, and then an Unna bandage to the neck, the forehead and the shoulders. The rationale is 0 that of splinting, warmth and protection from external trauma (e.g. squeezing) and additional external infection. Progressive healing follows and what is of more importance, it avoids recurrence, either locally or in remoter parts of the body such as about the kidney, in bone, or the ischio-rectal fossa. To reinforce the rest factor in addition to the Unna's paste bandage, a light plaster of Paris casing is applied over it, occasionally, and is retained for two to three weeks $\vec{\varphi}$ Results have been consistently good and patients have found it comfortable although they have expressed surprise at the radical treatment applied to what is regarded ordinarily as a triviag condition, a boil. It should be explained; that it is recurrent boils which are chiefly in mind, fox a surgeon seldom is asked to see a single primary boil.

\section{Cervical Adenitis.}

Both acute and chronic suppurating adenitis of the neck after opening or excision as need be, are treated by an Unna's paste bandage for several weeks.

\section{The Breast.}

Unna's paste bandages have proved of singular value in the treatment of two conditions of the breast, that of acute suppurative mastitis after pregnancy and that of delayed healing after radical excision of the breast for carcinoma.

Acute suppurative mastitis.-The main need in the treatment of suppurative mastitis is restoration of the general health, for these patients are always debilitated, the breast suppura- $₹$ tion being a local manifestation of this. The breast abscess is opened as it requires and the $\mathrm{o}$ edges of the skin incision are stitched back, so that there is ready exit for the pus and no need for a drain. The breast is encased in Unna's paste bandages which are supported from the shoulder and round the thorax. The nursing staff become enthusiastic on this treatment when they have seen one or two so treated, and enjoy applying a substantial and shapely Unna bras-or siere. The first is retained for two to three weeks. It is removed if the discharge has soaked $N$ through, and occasionally for the smell. The breast is then found healed but still swollen. A N second Unna's bandage is put on and retained for a fortnight by which time the mamma will be found comparable with its fellow. Women treated with one or more abscesses in the breast and have but two dressings in five to six weeks, from operation to recovery. Obviously the $\frac{Q}{\mathbb{D}}$ abscesses are opened thoroughly first under an anaesthetic. The rest which the infrequent $\stackrel{\Phi}{=}$ dressings bestow, the avoidance of expense to patients and hospital and early liberation of beds are lesser advantages. 
Carcinoma of the breast.-From time to time, after a thorough operation for excision of the breast, the skin flaps slough, leaving an area of variable size to be skin-grafted or to heal by granulation. If the latter method is chosen, exuberant granulations and pustular eczematous states of the surrounding skin are prone to develop and various dressings such as eusol and paraffin, red lotion, flavine, etc., are applied daily to overcome this. A single application of Unna's paste retained for ten to twenty-one days, dries this up and settles a problem which otherwise may drag on for months.

\section{The Abdomen.}

After acute appendicitis with general peritonitis, a sinus of the abdominal wall may persist. The application of a pad of Unna's paste plus an Unna's spica bandage will protect and splint the part while healing takes place beneath it.

\section{The Arm.}

Unna's bandages have been used frequently on the upper limb. The first case was in January I94I. A child aged 5 was scalded on the extensor surface of the arm on December Ist. He was admitted and dressed daily for $3 \mathrm{I}$ days, when he was discharged. He attended daily as an out-patient during January, at the end of which the area showed no signs of healing, and he was referred to the writer for a skin-graft. The area was about $3^{\prime \prime} \times 2^{\prime \prime}$, approximately elliptical. An Unna's paste bandage was applied. A week later the mother remarked gratefully on the improved behaviour and health of her son. After three weeks the bandage was cut off, it was hoped the area would be healed, but it was still half the size of the original lesion. It was Unna-ed again, and was discarded two weeks later when healing was complete. The contrast is 62 dressings in 62 days (it may have been oftener while he was an in-patient) with no improvement and deterioration in general health, against two dressings in 35 days and healing.

For repeated boils on the fore-arm or hand, and for whitlows which have been opened and the pyrexia has settled, an Unna's paste bandage is invaluable. It is retained for a fortnight. Similarly after a whitlow is healed, a patient may be referred for massage on account of the swelling, induration and stiffness of the part. Application of an Unna's paste bandage has been found to meet such patient's needs.

Sore elbow.- Unfortunately it is frequent now-a-days; to see a painful swollen or suppurating elbow after an injection for uro-selectan or pentothal or of blood or saline. In fact repeatedly have arms been unhealed, painful and incapacitating, weeks and months after the thyroid, the gall-bladder, the stomach or kidney lesion, etc., is well and forgotten. These conditions are a reproach to modern technique. A small incision over the area is indicated and then a stout Unna's paste bandage with the limb in position of $90^{\circ}$ flexion is applied. In two to three weeks healing is complete, the induration disappears, mobility gradually returns and the pain subsides.

\section{Burns and Scalds.}

For extensive burns and scalds on the abdomen, buttocks and thighs and limbs, when the gentian violet coagulation or whatever means have been used has come away and a granulating area remains, treatment by Unna's paste bandages applied at first for one day, then on alternate days and so on, extending the period as much as possible is successful. Progressive painless healing follows. All surgeons are familiar with the emotional instability and debilitation which follows frequent dressings. Unna's paste bandages are the patient's friend in avoiding these states which are distressing alike to the patient and to the nursing staff.

\section{Compound Fractures and War Wounds.}

It is well known that wounds fill up well under plaster of Paris casings and that fractures unite but it is also true that the skin doesn't heal well. A combination of an Unna casing plus plaster of Paris externally will give suitable conditions for epithelialisation.

\section{Conclusion}

Various conditions are described where Unna's paste bandages have been used with shortening of the period of healing, avoidance of pain, time and expense. Based on this successful experience this report is made with a plea for their wider use. 